\title{
UNA NUEVA CIUDAD PÚNICA EN HISPANIA: TGLYT - RES PUBLICA TAGILITANA, TÍJOLA (ALMERÍA)
}

\author{
POR \\ CARMEN ALFARO ASINS \\ Museo Arqueológico Nacional de Madrid
}

\begin{abstract}
RESUMEN
Entre los materiales arqueológicos que ingresaron en el M.A.N. de las antiguas excavaciones de L. Siret en Villaricos (Almeria), cabe destacar unas monedas de cobre con topónimo neopúnico, desconocidas hasta ahora, que evidencian la existencia de una nueva ceca púnica. La transcripción de dicho topónimo neopúnico en THLYT nos lleva a identificar esta ciudad emisora de moneda con la res publica Tugilitana, citada en una lápida del siglo II d. C. hallada en el término municipal de Tijola (Almeria). El extraordinario parecido fonético entre el nombre púnico, el latino y el de la localidad actual, facilita esta atribución. La factoría púnica podria estar emplazada en la Muela del Ajo de Tijola, importante yacimiento en el que se han documentado materiales arqueológicos que muestran una ocupación entre los siglos v y $\|$ a. C.
\end{abstract}

\section{SUMMARY}

This article deals with some coins which came into the Museo Arqueológico Nacional among the archaeological materials found in the ancient excavations conducted by L. Siret in Villaricos (Almeria). They are copper coins with Neopunic place-name and they show the existence of a new Punic mint up to now unknown. The transcription as TGLYT induces us to identify this town issuing coins with the res publica Tagilitana quoted in a II century A. D. commemorative tablet found in the area around Tijola (Almeria). The extraordinary similarity between the Punic, Latin and present-day name, makes the attribution easier. The location of the Punic factory should be La Muela del Ajo in Tíjola, an important archaeological deposit occupied between $\mathrm{v}$ and II centuries B.C.

\section{INTRODUCCIÓN}

Las acuñaciones púnicas de Hispania pertenecen en razón de su autoridad emisora fundamentalmente a dos categorias políticas. La primera autoridad, foránea se corresponde con las emisiones provinciales de carácter militar realizadas por los cartagineses en la Peninsula entre el 237 y 206 a. C. La segunda, de carácter local, a una serie de ciudades de sustrato fenopúnico que acuñan bajo su propia autoridad municipal utilizando el alfabeto fenicio, el elemento más determinante de su filliación sea cual sea su estadio evolutivo.

La cronologia de estas emisiones púnicas municipales, en general, se puede situar entre principios del siglo III a. C. y la primera mitad del siglo I d. C. ( 54 d. C.), aunque no todas las cecas acuñaron durante este largo período, ya que la importancia de cada ciudad, sus necesidades o su especial status en época romana favoreció o no la emisión de moneda. En la mayoria de los casos aún existen grandes problemas para fijar la cronología absoluta de muchas de estas emisiones y tanto las excavaciones arqueológicas como los nuevos hallazgos monetales han de ir refrendando las fechas que se proponen en cada caso.

Estas ciudades emisoras púnicas de Hispania se sitúan en general en la parte meridional de la Península y la isla de Ibiza, aunque su área de desarrollo es más amplia de lo que en principio se suponia, pues no son costeras, sino que también están situadas mucho más al interior, llegando incluso algunas como Turiregina y también posiblemente Arsa a estar ubicadas en la provincia de Badajoz.

Muchas de estas ciudades son antiguas fundaciones fenicias y colonias púnicas bien conocidas por las fuentes literarias y arqueológicas, generalmente costeras y con puertos de importancia como Gadir (Cádiz), Ebusus (Ibi- 
za), Malaca (Málaga), Abdera (Adra, Almeria), Seks (Almuneecar, Granada), Olontigi (Aznalcazar, Sevilla), Ituci (Tejada la Nueva, Huelva) y, con mayores dudas en la identificación, Albata (Abla, Almeria), cuyas monedas en general se caracterizan por una escritura normalizada púnica o neopúnica, tipos helenizados y volúmenes de emisiones aceptables.

Otras ciudades generalmente situadas más al interior, posiblemente asentamientos más recientes de sustrato mayoritariamente africano, son Asido (Medina Sidonia, Cádiz), Bailo (Bolonia, Cádiz) y otras menos conocidas como Lascuta (Alcalá de Gazules, Cádiz), Iptuci (Prado del Rey, Cádiz), Oba (Jimena de la Frontera, Cádiz), Turiregina (Casas de Reina, Badajoz), Arsa (¿Azuaga?, Badajoz)', y Vesci
(¿Gaucin?, Málaga, o quizás mejor al Sur de la provincia de Badajoz). Todas ellas, denominadas convencionalmente "libio-fenicias» desde que Zobel en 1863 les dio ese nombre, presentan monedas con una escritura neopúnica no normalizada en ocasiones aberrante, tipos menos influidos de la plástica grecorromana y salvo alguna excepción, volúmenes menores de emisión y en algunas cecas coyunturales.

A estos dos grupos cabe añadir otra serie de monedas púnicas «inciertas» que presentan tanto epigrafia púnica como neopúnica, normalizada o no, pero que por el momento no se pueden situar con seguridad en ningún emplazamiento conocido, existiendo la posibilidad, incluso, de que algunas fueran monedas africanas ${ }^{2}$. También cabe incluir, como excepción,

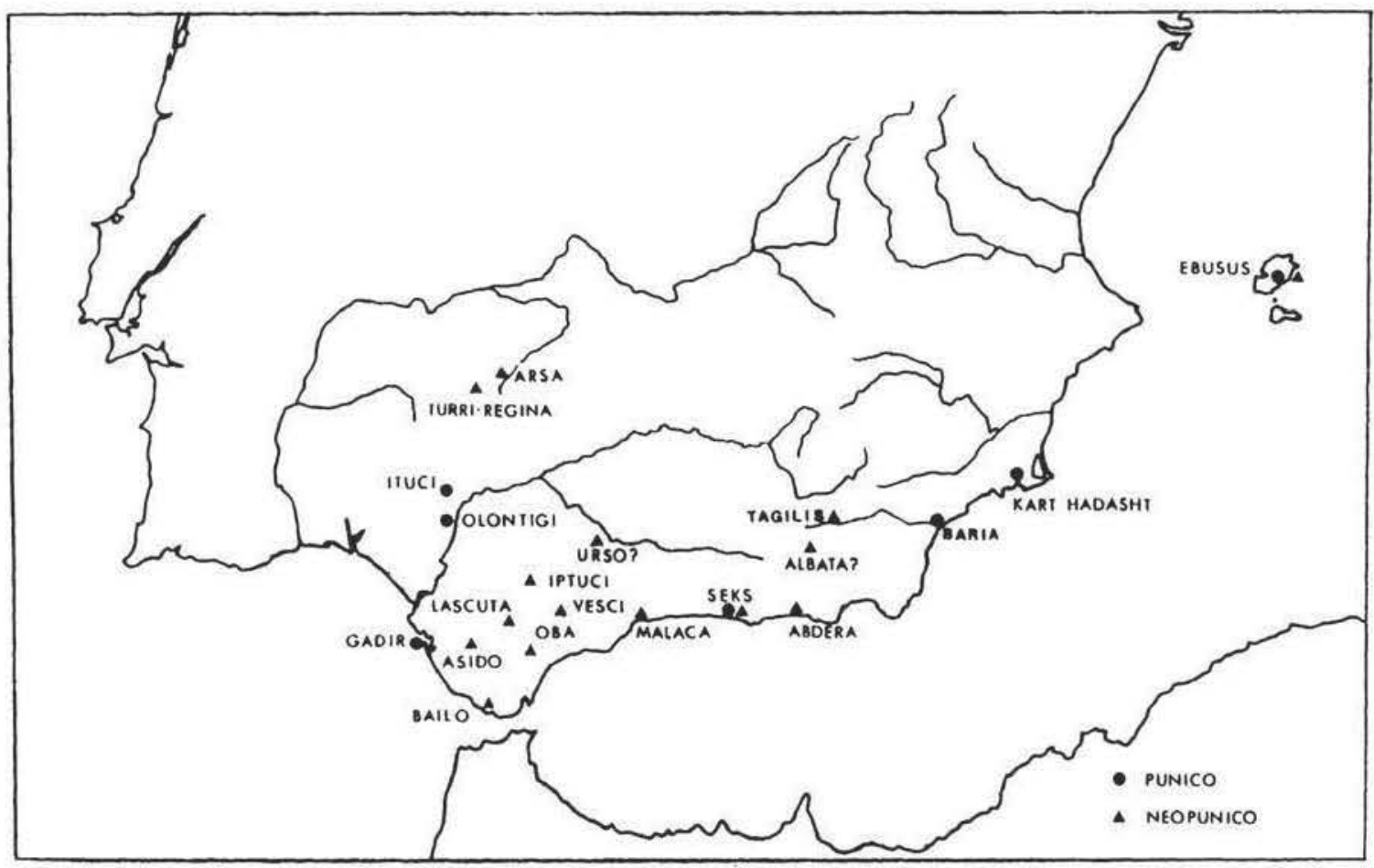

Figura 1.-Localización geográfica de las cecas feno-púnicas hispanas.

I Sobre la localización de esta ceca recientemente ha tratado M. P. García-Bellido: Sobre la dos supuestas ciudades de la Bética llamadas Arsa. Nuevos testimonios de púnicos en la Baeturia Turdula, Anas (en prensa), quien definitivamente la ubica en la zona de Badajoz y descarta la existencia de la supuesta Arsa gaditana.

\footnotetext{
2 La problemática de estas monedas y las atribuciones que se han barajado en C. Alfaro Asins: Epigrafia monetal púnica y neopunica en Hispania. Ensayo de sintesis, Glaux, 7. (Ermanno A. Arslan studia dicata), Parte I, Milano, 1991. págs. 137-144 (en adelante citado Alfaro Asins, 1991).
} 
la ceca de Baria (Villaricos), cuyas monedas fueron emitidas por gentes punicas aunque no presenten epigrafes.

Este sustrato semita lo encontramos también en muchas otras cecas de la zona meridional de la Peninsula que no consideramos «púnicas" como, por ejemplo, Carmo, Obulco o Ilipa, pues sus monedas presentan una iconografia y simbolos fenicio-púnicos, en ocasiones bajo formas grecorromanas, como ha puesto de manifiesto fundamentalmente Garcia-Bellido, aunque la epigrafia que utilizan es ibérica o latina. En este sentido cabe citar también que la cerca de Ursone (Osuna) parece incluir una letra yod en su primera emisión, aunque no por ello se ha considerado púnica ${ }^{3}$.

Pues bien, una vez realizada esta breve introducción sobre las ac.ñaciones púnico-hispanas, nos cabe la satisfacción de poder incluir una nueva ceca municipal: Tagilit (Tijola, Almeria), que pasamos a comentar ${ }^{+}$.

\section{EL MATERIAL NUMISMÁTICO DE VILLARICOS}

Entre 1931 y 1935 ingresaron en el Museo Arqueológico Nacional una serie de materiales procedentes de las excavaciones arqueológicas efectuadas por Luis Siret en la antigua Baria (Villaricos, Almería), fundamentalmente en la necrópolis, entre finales del siglo pasado y los primeros años de este 5 . Además ingresaron 51 diarios de excavación, su biblioteca y otros manuscritos con el estudio y la descripción de muchos de estos materiales, entre ellos las monedas. Con posterioridad a estas fechas cabe destacar los trabajos de M. Astruc ${ }^{b}$ y las exca-

J. M. Solá Solé: Miscelánea púnico-hispana III, Sefurad, XXV, 1965, págs. $39-43$, atribuye a esta ceca. por la lectura del topónimo púnico, una serie de monedas consideradas "inciertas".

4 Inicialmente dada a conocer en el homenaje a don Leandro Villaronga Garriga. Cfr. C. Alfaro Asins: Tagilit, nueva ceca púnica en la provincia de Almeria, Acta $\mathrm{Nu}$ mismática (en prensa).

' L. Siret: Villaricos y Herrerias, antigüedades púnicas, romanas, visigóticas y árabes, Memorias de la Real Academia de la Historia, XIV. 1909, págs. 380-478.

- M. Astruc: La necrópolis de Villaricos, Madrid, 1951. (En adelante citado Astruc.) vaciones que desde 1974 viene realizando $M$. J. Almagro en el yacimiento?

El material numismático de estas antiguas excavaciones que ha llegado a nosotros tiene un gran interés, aunque la mayoria de las piezas no sabemos exactamente en qué situación se hallaron dentro del yacimiento. Algunas de estas monedas sin contexto conocido fueron descritas por J. M." Vidal que, inicialmente, publicó nueve divisores de la ceca de Baria hasta ese momento desconocidos, después 82 monedas de cobre de gran peso de la misma ceca y, por último, describió otras 157 monedas halladas en el yacimiento, de ellas 85 de Baria, las mismas 82 anteriores a las que añadió otros tres nuevos ejemplares, los ya citados nueve divisores de Baria, dos monedas púnicas, 13 ibéricas, siete hispano-romanas, ocho romanas republicanas y 33 imperiales .

Además de estas monedas sin contexto recogidas por Vidal, hemos podido clasificar 105 nuevas piezas, aunque se han desestimado otras 188, prácticamente frustras. Por último, el lote más interesante lo forman 143 monedas que conservan el importante dato de la tumba en que se hallaron y que daremos a conocer próximamente.

Entre todo el material numismático procedente de Villaricos, desde nuestro punto de vista, destacan una serie de monedas de tipo púnico

7 Arqueologia 79. Memoria de las aciuaciones programadas en el año 1979. Madrid, 1979. pág. 37, n. 25. Arqueologia 80. Memoria de las actuaciones programadas en el año 1980, Madrid, 1981, pág. 38, n. ${ }^{\circ} 25$. Arqueologia 83 . Memoria de las actuaciones programadas en el año 1983. Madrid, 1984, pág. 14, n." 1.3. M. J. Almagro Gorbea: La necrópolis de Baria (Almeria). (ampañas de 1975-78. Madrid, 1984. M. J. Almagro Gorbea: La necrópolis de Villaricos, campaña de 1983 (en prensa). De las excavaciones de este último año cabe destacar un tesorillo de 19 monedas de Cástulo halladas junto a una de Gadir. Cfr. M. J. Almagro Gorbea: Un tesorillo de monedas ibéricas y púnicas de la antigua Baria, Cuadernos de Prehistoria de la Universidad de Granada, 11, 1986. págs. 331-353.

* J. M.* Vidal Bardán: Moneda inédita de Baria, Acta Numismática, IX, 1979, págs. 37-39.- Id: La circulación monetaria de Villaricos: La ceca de Baria, II Simposi Numimàtic de Barcelona, Barcelona, 1980, págs. 151-155. Id: : La circulación monetaria de Villaricos según los fondos del Museo Arqueológico Nacional, Museos-2, 1982. págs. 15-23. 

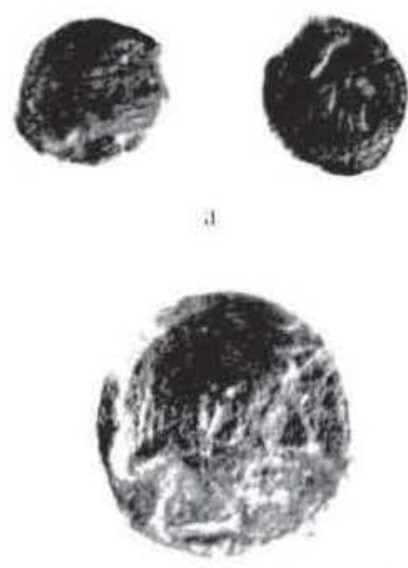

Figura 2. Morredas de Tagalit del tipo 1.

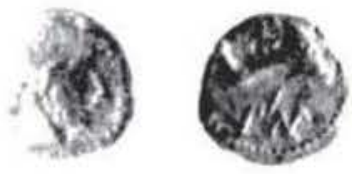

b

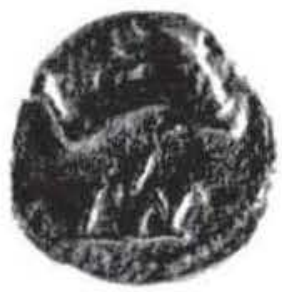

Los dos ejemplares que conocemos de este primer tipo de monedas presentan una conservación muy deficiente, aunque nos parece observar un mismo cuño de anverso y claramente distintos cuños de reverso, diferenciables tanto en la forma y tamaño del delfín como en la leyenda.

Los pesos de las dos monedas que conocemos son de 2,30 y $1,87 \mathrm{gr}$, lo que parece evidenciar que el valor de estas piezas seria el de cuartos de una unidad tanto de $8-9$ gr como de 10-11 gr, aunque por la tipologia que presentan nos inclinamos a pensar que siguen el último, más reciente y que en otras cecas como Gadir presenta divisores tipológicamente exactos a éstos. Es pues lógico deducir que estas pequenas monedas fueran divisores de otras de mayor tamaño que o bien no se acuñaron, no han Ilegado a nosotros o no somos capaces de relacionar entre si.

La tipologia, el reducido tamaño e incluso la leyenda de estas monedas recuerdan fundamentalmente algunos divisores de Olontigi con cabeza masculina y delfin a derecha ${ }^{10}$, aunque estos mismos tipos de anverso y reverso son frecuentes tambièn en otros talleres púnicos de Hispania como Abdera ${ }^{11}$, Seks ${ }^{12}$, Gadir ${ }^{13}$ y

10 A. Vives y Escudero: La moneda hispanica, Madrid, $1926,1 \times X X X \mid X-4$ y 7 (en adelante citado Vives)

i) Vives, $L X X X 1-8$

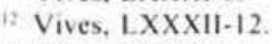

11 Vives, LXXIV-11. 
Asido ${ }^{14}$, del Norte de África como Iol Caesarea ${ }^{15}$, asi como también utilizados en otras cecas del sur de la Península ${ }^{16}$ y cuadrantes ibéricos ${ }^{17}$.

Esta similitud tipológica con monedas púnicas de otras cecas bien conocidas y su reducido tamaño han podido ser quizás las causas de que no conozcamos más ejemplares de esta nueva ceca que, si existen en otras colecciones, se han podido clasificar con el numerario de alguna de las ciudades citadas, fundamentalmente Gadir y Olontigi, como sucedió con la pieza $n^{\circ} 1$ atribuida erróneamente a Gadir por Vidal Bardán.

Sin embargo, creo poder identificar estos ejemplares con una moneda recogida primero por Delgado ${ }^{18} \mathrm{y}$ después por Vives entre las «inciertas» (figura 3). Este último investigador ya no vio directamente la pieza por lo que se limitó a reproducir y describir el dibujo de la obra de Delgado sin transcribir la leyenda comentando sólo que quizás fueran una moneda de Gades mal vista ${ }^{19}$.
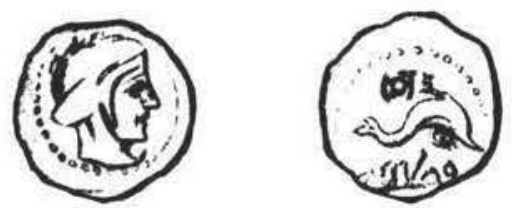

Figura 3.- Moneda "púnica no clasificada" de Delgado (lám. LXXXV, n. ${ }^{\circ}$ ) o "incierta" de Vives (lám. CXX, n. ${ }^{\circ} 8$ ).

it G. K. Jenkins: Sylloge Nummorum Graecorum. The Royal Collection of Coins and Medals National Museum. North Africa Syrtica-Mauretania, Copenhagen, 1969. n. ${ }^{\circ}$ 106. (En adelante citado SNGCop.)

is J. Mazard: Corpus Nummorum Numidiae Mauretaniaeque, París, 1955, núms. 566 y 567. (En adelante citado Mazard.)

i6 Carteia, Vives, CXXVI, 11-13, CXXVII, 4 y 8. CXXVIII, 8 y 10. Salacia, Vives, LXXXIV-11.

i7 Vives, XXIII-4, XXIV 7-12, XXXI 7-10, XXXII 12 6, XXXIII 3-14, LXVII-5.

is A. Delgado: Nuevo método de clasificación de las medallas autónomas de España, Sevilla, 1873, Apéndices, págs. $387-388, n{ }^{\circ} 5, \mathrm{LXXXV}, 5$ (en adelante citado Delgado).

${ }_{19}$ Vives, pág. 120, n. ${ }^{\circ} 22, \mathrm{CXX}-8$.
Esta moneda recogida por Delgado y $\mathrm{Vi}$ ves que, al parecer, se conservaba en la colección de don Eduardo Navarro de Málaga, se describe en ambos autores con cabeza femenina con tocado especial en anverso, $y$, en reverso, el delfin con creciente y glóbulo, debajo se sitúa entre dos líneas de leyenda púnica. Este creciente con glóbulo que citan Delgado y Vives no es otra cosa, desde nuestro punto de vista, que la aleta pectoral mal interpretada.

La leyenda superior, según Rodriguez de Berlanga, estaria formada por cuatro letras que transcribe al hebrero en mem, beth, arin y lamed, $y$ es igual a la que se encuentra in monedas de Gadir, Seks, Tingis y Lixus; es decir, se trataria de la conocida fórmula $\mathbf{M B} \mathbf{B}^{\prime} \mathbf{L}$ o $\mathbf{M P} \mathbf{P}^{\prime} \mathbf{L}$, según interpretaciones, como también se desprende de la grafia de los signos del dibujo. La leyenda inferior, a su juicio, es de seis letras que transcribe en: la primera una 'ayin, la segunda una taw, la tercera una lamed, la cuarta una nun, la quinta duda entre beth-daleth-zayin y la sexta una gimel ('TLN B-D-Z G) ${ }^{20}$.

En general la mayoria de los numismáticos desde L. J. Velázquez han tendido a transcribir la segunda letra de esta leyenda por una beth: MB'L, con significado "de los ciudadanos", «del pueblo de ${ }^{21}$. Por su parte los epigrafistas, siguiendo a F. Pérez Bayer, se han inclinado por la trascripción con peh: MP'L, con significado de «obra», «trabajo» y por extensión "acuñación de»" ${ }^{22}$. Desde un punto de vista estrictamente formal de la letra según aparece en

\footnotetext{
${ }^{20}$ Delgado, págs. $387-388$, n. $^{\circ} 5$.

21 L. J. Velázquez: Ensayo sobre los alfabetos de las letras desconocidas, que se encuentran en las más antiguas medallas y monumentos de España, Madrid, 1752. Uno de los numismáticos que siguen esta lectura es $\mathrm{E}$. Acquaro: Note di epigrafia monetale punica - I, Rivista Italiana di Numismatica, LXXVI, 1974, 77-82. E. Acquaro: Le monete neo-puniche di SKS. Nota epigráfica, Studi per Laura Breglia. Supplemento del Bollettino di Numismatica, 4, 1987, 235-237.

22 F. Pérez Bayer: Del alfabeto y lengua de los fenices, $y$ de sus colonias, Madrid, 1772. Más recientemente defendido por J. M. Solá Solé: A propósito de un rótulo monetario. Miscelánea púnico-hispana IV, Sefarad, XXVII, 1967, págs. 16-28, y G. K. Jenkins: Spain-Gaul, Sylloge Nummorm Graecorum. The Royal Colection of coins and medals Danish National Museum, MunksgaardCopenhagen, 1979, n. ${ }^{\circ} 7,13-33$ y 54-60. (En adelante citado SNGCop Spain.)
} 
las monedas de Gadir fundamentalmente, insistimos en que la grafia corresponde a la de una peh punica, con independencia del significado de la palabra con una u otra letra.

Esta leyenda superior no es visible en ninguna de las monedas que presentamos, aunque es muy posible que este rótulo exista y contenga la conocida förmula $\mathbf{M P} \cdot \mathbf{L}$, habitual en algunas cecas púnicas hispanas y norteafricanas, con sentido de «acuñación de». Por otra parte, esta hipótesis se afianza en el convencimiento de que estas monedas son similares a la recogida entre las inciertas por Delgado y Vives, antes citada, que se reproduce con esta leyenda superior, claramente dibujada e interpretada.

En cuanto a la leyenda inferior, que es la que hemos podido observar directamente en los ejemplares hallados en Villaricos, cabe destacar que se trata de una epigrafia normalizada $\sin$ apenas problemas de lectura (figura 4 ). El primer signo es una clara letra taw neopúnica

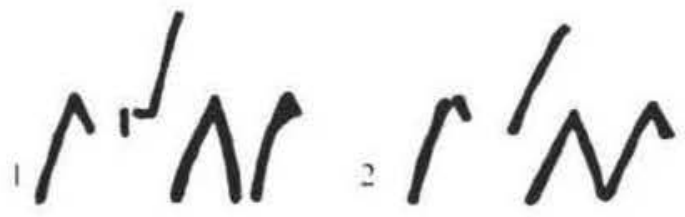

Figura 4.-Leyendas de las monedas del tipo 1: TGLT

similar a la que aparece en Olontigi ${ }^{23}$, en algunas piezas de Abdera ${ }^{24}$, en divisores tardios de Gadir $^{25}$ y en abundantes cecas norteafricanas. Esta letra en la primera moneda, la de mayor peso, presenta un trazo muy grueso en su parte superior por lo que pudiera parecer la cabeza cerrada de la letra, a la manera de una beth en negativo (figura 4.1).

La segunda letra es una también clara gimel neopúnica, en ambas monedas representada por un doble trazo angular de igual longitud ${ }^{26}$. Este

23 J. M. Solá Solé: Acuñaciones monetarias de Olontigi, Numisma, 74, 1965, pág. 23, lám. 1.

24 SNGCop Spain, n. 69.

25 C. Alfaro Asins: Las monedas de Gadir/Gades, Madrid, 1988, pág. 60, leyendas núms. 3.1. 3.2 y pág. 62 , leyenda anómala $n .^{\circ} 8$. (En adelante citado Alfaro.)

${ }^{26} \mathrm{~F}$. Friedrich, y W. Rollig: Phönizisch-punische Gramatik, Roma, 1970, núms. 45, 46 y 47. (En adelante citado Friedrich.) timo de gime/ también lo encontramos en algunas monedas de Olontigi ${ }^{27}$, en Ebusus ${ }^{2 *}$ y en algunas cecas norteafricanas.

El tercer signo es una lamed con distinta grafia en cada moneda. En el primer ejemplar la letra adopta una grafia púnica pero trazada en negativo, forma errónea similar a la que documentamos en una moneda de la primera serie de Gadir ${ }^{20}$ (figura 4.1). En la segunda pieza la letra adopta una forma claramente neopúnica que sobresale por encima del resto de la leyenda (figura 4.2), grafia también documentada en monedas tardias de Gadir ${ }^{30}$, Seks ${ }^{31}$, Mala$\mathrm{ca}^{32}$ y Olontigi ${ }^{33}$.

La cuarta letra parece ser otra taw de grafia similar a la anterior, aunque no se aprecia claramente en el primer ejemplar y en el segundo los trazos que la forman no están unidos por el vértice, como es habitual.

Es posible que pueda haber algún otro signo a continuación de los reseñados como opinaba Rodriguez de Berlanga, aunque no parece probable dado el importante relieve de los trazos que se conservan y el escaso campo monetal que queda libre hasta el borde de la pieza.

Por lo expuesto, la transcripción TGLT que proponemos es bastante clara, incluso también aplicable a los tres primeros signos del dibujo de la obra de Delgado, después recogido por Vives. Esta leyenda podría vocalizar en TaGyLaT o TaGyLiT, con una estructura similar al 'BDRT o ABDeRaT de la cercana ceca de Abdera.

Asi pues estas monedas tanto por tipologia, como por metrologia y sobre todo por presentar la leyenda neopúnica MP'L / TGLT, están en la linea de las acuñaciones municipales que

\footnotetext{
27 SNGCop Spain, n.. 167.

2 En Easi todos los reversos del grupo XIX de M. Campo, Las monedas de Ebusus, Barcelona, 1976.

24 C. Alfaro Asins: Variante en moneda de Gadir, $\mathrm{Nu}$ misma, 177-179, 1982, págs. 59-63.

19 Alfaro, pág. 60, leyenda n. 3.2 y pág. 61, D.

3 C. Alfaro Asins: Observaciones sobre las monedas de Seks según la colección del M.A.N., Almúñecar, Ar. queologia e Historia, III, 1986, págs. 75-103.

$12 \mathrm{M}$. Campo: Algunas cuestiones sobre las monedas de Malaca, Aula Orientalis, 4, 1986, pág. 144.

"1 J. M. Solá Solé: Acuñaciones monetarias de Olontigi, citado, pág. 23, lám. I.
} 
conocemos en otras ciudades punico-hispanas. fundamentalmente Gadir. Por comparación con las series de la citada ceca y por la epigrafia neopúnica aunque normalizada de estas monedas. podemos situar su emisión entre finales del siglo III y mediados del II a. C.

\section{Tipo 2}

Anv.- Palmera sin frutos.

Rev.- Creciente, encima estrellas de 4 rayos y debajo leyenda punica TGYLT (..?)

\section{I $3.37 \quad 18.00$ - RC Archivo Villa- ronga (fig. 5a). \\ $\begin{array}{llllll}2 & 3,05 & 18,00 & 12 & R C & \text { MAN, Siret }\end{array}$ Villaricos, $8^{\text {is }}$ (fig. 5b).}
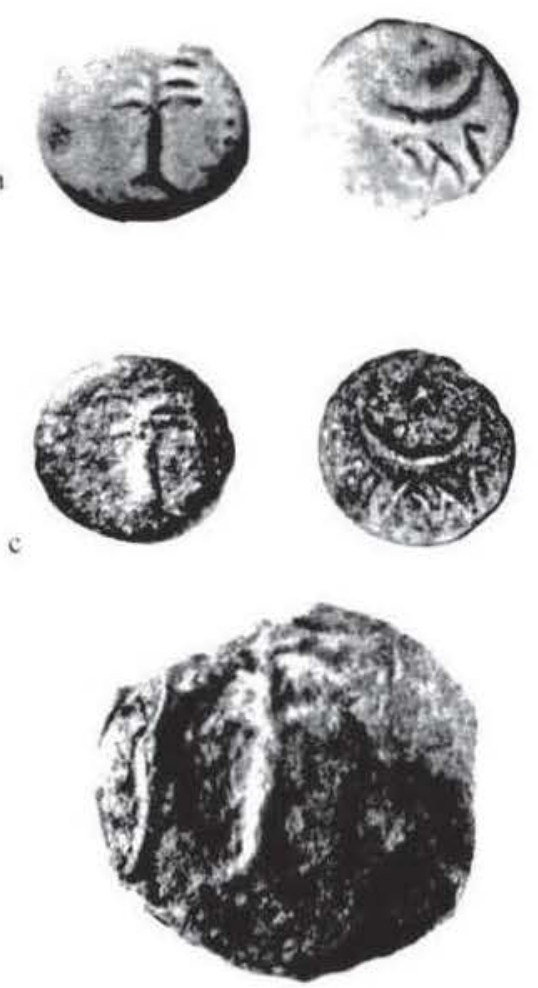

b $(\times 2)$

\section{$33.00 \quad 18.00$ 9 $R C$ IVDJIX-10-9. Giomer More- no ${ }^{36}$ (fig. $5 \mathrm{c}$ ). \\ 42.8417 .0016 RC MAN, Siret \\ Villaricos. tumba 937 \\ (fig. 5d).}

De las cuatro monedas que actualmente conocemos de este tipo. las dos primeras que estudiamos, conservadas en la colección del M.A.N. proceden de Villaricos y una de ellas, la $n . " 4$, se halló como único material numismático en la tumba n. ${ }^{\circ} 937$. Esta tumba corresponde a un enterramiento colectivo dentro de una gran camara hipogea de forma rectangular con corredor de acceso, excavada en la colina denominada El Garban/al, dentro de la zona
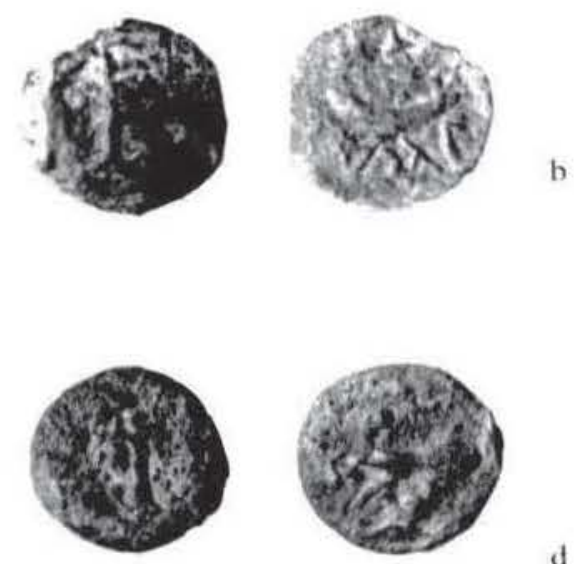

d

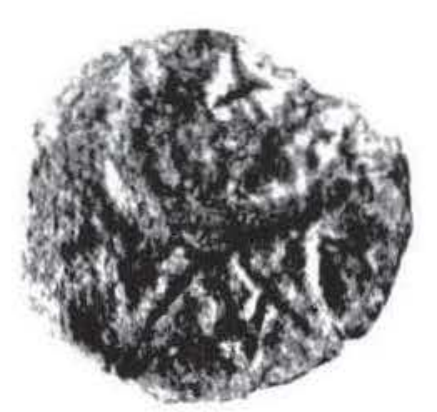

Figura 5. Monedas de Tagilit del tipo 2.

${ }^{4}$ Esta moneda, sin procedencia conocida, nos ha sido amablemente proporcionada por el doctor Villaronga.

is Al parecer hallada en los alrededores de los hipogeos. is Agradecemos a la doctora Garcia-Bellido tanto las sugerencias que ha realizado a este trabajo como la ficha de la moneda del Instituto de Valencia de don Juan, colección numismática que catalogó gracias a una beca de la Fundación March. 
de Los Conteros. Este tipo de hipogeos albergaban inicialmente inhumaciones pero a partir del siglo II a. C. se suelen reutilizar por lo que contienen tambien incineraciones ${ }^{17}$. Concretamente en el hipogeo n." 937 se hallaron. además de la moneda, un huevo de avestruz entero sin decorar. una lampara rodia. copitas de barro, un amuleto de barro con forma de racimo. un anillo de plata, estrigilas de hierro, varillas y aros de bronce, clavos y espiguillas circulares de hueso adornadas con trazos radiados incisos, asi como restos de unos catorce esqueletos sin quemar ${ }^{3 x}$.

En general. los materiales de estos hipogeos de Villaricos son datables entre los siglos IV a. C. y 1 d. C... aunque es dificil diferenciar los ajuares de cada enterramiento debido a los revueltos provocados por las reutilizaciones, los antiguos saqueos y los derrumbes de los techos de las cámaras ${ }^{14}$.

En cuanto a metrologia las cuatro monedas que conocemos tienen un peso de 3,37, 3,05. 3,00 y $2,84 \mathrm{gr}$, con una media de $3,065 \mathrm{gr}$, por lo que podrian ser tanto mitades de poco peso del sistema de 8-9 gr como, mejor, cuartos del patrón de $10-11 \mathrm{gr}$. Dada la similitud tipológica con los divisores de Baria, nos preguntamos si no podria tratarse también de una misma denominación aunque más ligera. La posible pérdida de peso de las monedas de Tagilit quizás pudiera deberse al predominio del plomo en su composición $^{20}$. Por el contrario en los divisores de Baria predomina el cobre ${ }^{41}$.

\footnotetext{
17 Astruc, pág. 67. tipo J.

4x Fstos son los materiales que de manera imprecisa y aislada cita Astruc, págs, 70, 71, 72, 76, 77, 78, 79 y 80. En los diarios de excavaciones de Siret, redactados por su capataz Pedro Flores, figuran aproximadamente las mismas piezas con algunas variaciones en las denominaciones o interpretaciones. La identificacion de los materiales de cada tumba y el estudio detallado de la necropolis de Villaricos está, como se puede observar, por hacer.

iv M. J. Almagro: Las excavaciones en la necrópolis púnica de Villaricos, Homenaje a Luis Siret (1934-1984). Sevilla. 1986, págs. 629-632.

41" El análisis metalográfico de dos monedas ha dado un alto porcentaje de plomo del $56-57 \%$, seguido del cobre con el $38 \%$, estaño con $3.5 \%$, hierro con $0.10-0.20 \%$, niquel con $0,13-0,19 \%$, antimonio con $0,08-0,09 \%$ y plata $\operatorname{con} 0,017-0,067 \%$.

+ El análisis de una moneda ha proporcionado un $79.80 \%$ de cobre, $12.22 \%$ de plomo, $7.55 \%$ de estaño, $0.219 \%$ de antimonio, $0.046 \%$ de plata y $0,03 \%$ de hierro.
}

Como en el caso anterior, las monedas presentan una conservación deficiente, aunque es posible apreciar su tipologia y leyenda, por lo que parecen proceder de un mismo par de cuños.

Tipológicamente estas monedas están en la linea de las emisiones cartaginesas, ya que la palmera es después del caballo el tipo más representado y, en especial. son muy proximas a las acuñaciones punicas de la antigua Baria, de donde quizás tomaran el modelo. Como tipo principal y casi siempre representada con frutos, la palmera es muy frecuente en emisiones cartaginesas de plata y bronce de Sicilia ${ }^{42}, y$ de oro y bronce de Cartago ${ }^{4}$. En la Península Iberica la palmera como tipo principal la encontramos en los reversos de la ceca de Baria, tanto en el valor superior ${ }^{44}$ como en su divisor. cuarto del anterior ${ }^{+5}$. También aparece en los reversos de dos emisiones de bronce hispanocartaginesas, aunque en todos estos casos se representa con frutos ${ }^{\text {th }}$.
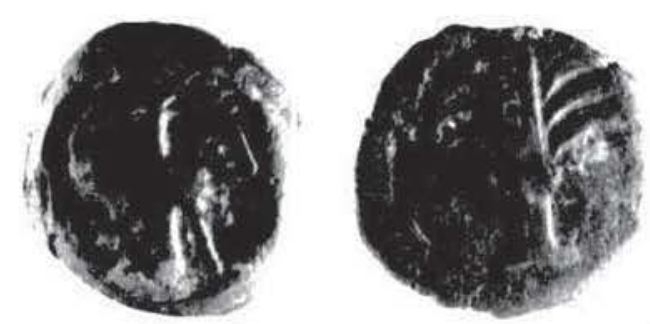

a
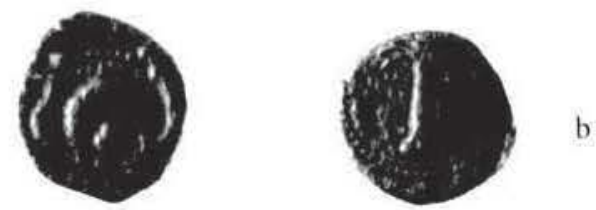

Figura 6.- Unidad y divisor de Baria.

22 SNGCop, nums, 70-72, 73-74, 102-106, 107-108. $124-125,126-127$.

"SNGCop. nums, 130, 132-133 y 415 . Una excepcional y nueva moneda de oro con reverso palmera descrita en L. Mildenberg: Punic Coinage on the eve of the First War Against Rome, a reconsideración. Studia Phoenicia X, Punic Wars, leuven, 1989, pág. 12.

${ }^{*}$ L. Villaronga: Las monedas hispano-cartaginesas, Barcelona, 1973, Clase XII. (En adelante citado Villaronga.)

4 J. M. Vidal: Moneda inédita de Baria, citado, páginas $37-39$.

th. Villaronga, Clase VIII. Tipo I, Grupo III A n. ${ }^{\circ} 113 \mathrm{y}$ Clase X. Tipo II, nums. 126 y 127. 
El tipo de reverso, un creciente con estrella de cuatro rayos, está en evidente relación con el uraeus con creciente y astro que aparece en los anversos de los divisores de Baria que además presentan también, como hemos dicho ya. una palmera en reverso.

El creciente, con o sin astro, como tipo secundario es muy frecuente en el numerario hispánico y norteafricano, especialmente en emisiones de tipo púnico, pero es muy raro como tipo principal. En la Península Ibérica como tipo principal, al menos hasta hace poco, sólo lo utilizaba la ceca de Malaca en los anversos de unas escasas monedas de bronce cercanas al cambio de Era ${ }^{47}$ (figura 7a). El creciente con estrella lo encontramos también en el reverso de unas monedas de bronce de unos $3,50 \mathrm{gr}$ con el topónimo BALLEIA, desconocidas hasta hace poco, que se adscriben a la zona sur de Badajoz ${ }^{48}$ (figura $7 b$ ). Esta nueva moneda con tipologia púnica aunque con rótulo latino de la Baeturia Turdula, viene a sumarse a las cecas de Turiregina y Arsa para confirmar la presencia de gentes feno-púnicas en la zona de Badajoz.

a
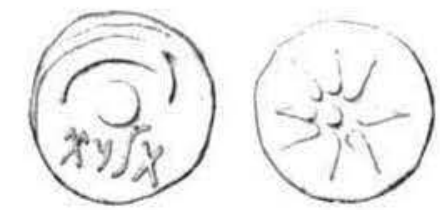

b
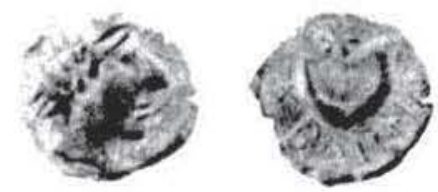

Figura 7.-Monedas de Malaca y Balleia con creciente como tipo principal.

\footnotetext{
47 Vives. LXXXV-5. M. Campo: Algunas cuestiones sobre las monedas de Malaca, citado, Periodo IV. Posi c. 45 a. C. Serie V11 ${ }^{\mathrm{a}} \mathrm{B}$.

th Una de 3,06 gr hallada en el poblado de Hornachuelos (Badajoz) según F. J. Jiménez Ávila: Estudio numismático del poblado de Hornachuelos (Ribera del Fresno. Badajoz), Series de Arqueologia Extremeña, n." 4. Cáceres, 1990, $n{ }^{\circ}$ 180: en pág. 92 comenta el hallazgo de más monedas similares en el yacimiento. Otro ejemplar de $3.78 \mathrm{gr}$ publicado recientemente en el Catálogo de la subasta de Jesús Vico realizada el 3 de marzo de 1993, n." 39.
}

Recientemente también se están dando a conocer una serie de pequeños divisores de plata que aparecen, fundamentalmente, en contextos de la Segunda Guerra Púnica en el Levante peninsular ${ }^{+0}$. Algunos presentan un creciente con astro como tipo de reverso ${ }^{50}$ (figura 8 a). otros que parecen ser sus divisores, conocidos gracias al importante hallazgo de Villarrubia de los Ojos (Ciudad Real), presentan el creciente y un delfín a derecha entrando en él ${ }^{\text {" }}$ (figura 8b). Por último otros divisores, también desconocidos hasta su hallazgo en Camarasa (Lérida), presentan una cabeza masculina en anverso y un creciente con punto en reverso (figura $8 \mathrm{c}$ ) o también una cabeza femenina en anverso y creciente con punto entre dos delfines en reverso (figura $8 \mathrm{~d})^{5 ?}$.

En el Norte de África el creciente con o sin astro aparece en el reverso de algunas monedas punicas de Bulla Regia y Macomada ${ }^{57}$. El creciente con estrella de seis rayos, en la misma disposición que el de las monedas de Tagilit, lo encontramos también en ejemplares mucho más recientes de Juba II y Ptolomeo ${ }^{4}$.

En Italia este tipo principal de reverso lo encontramos exactamente igual que en Tagilit, es decir con estrella de 4 rayos, en monedas etruscas ${ }^{55}$. El creciente con o sin glóbulo central acompañado de estrellas también aparece en uncias semilibrales anónimas, acuñadas en

24 Para M. P. Garcia-Bellido: Las religiones orientales en la Peninsula lbérica: documentos numismáticos. I. AE.Arq. 64, 1991, pág. 48. figura 13, y Las relaciones económicas y la moneda entre Massalia, Emporion y Gades. Simposio Internacional sobre Griegos o lheros, ss. VI-IV, Ampurias, 1991 (en prensa), estas monedas de unos $0.38 \mathrm{gr}$ son agorah, valor citado en algunos textos antiguos que equivale a $1 / 20 \mathrm{del}$ shekel.

41 M. Garcia Garrido, y S. Costa: Divisores de plata con tipologia helénico-púnica, Acła Nimismática, 16, 1986. págs. 53-56. M. P. Garcia-Bellido: El tesoro de Mogente y su entorno monetal, Valencia, 1990, pags. 60-61, lam. V. n. 2 .

M. Garcia Garrido: El hallazgo de Villarrubia de los Ojos, Acta Numismática, 20, 1990, nums. 115-120.

52 M. Crusafont i Sabater: Dos hemiobols inedits de la zona del Segre, Acta Numismárica, 19. 1989, pảgs. 53-58.

53 Mazard, núms. 515-516 y 522.

4 Mazard, núms. 189-192, 299-300 y 504-505.

55 Para la ilustración de las monedas aunque no para la cronologia remitimos al clásico trabajo de A. Sambon: Les monnaies antiques de I\%alie, I. Paris, 1903-1904. núms, 60,65 y 67 . 

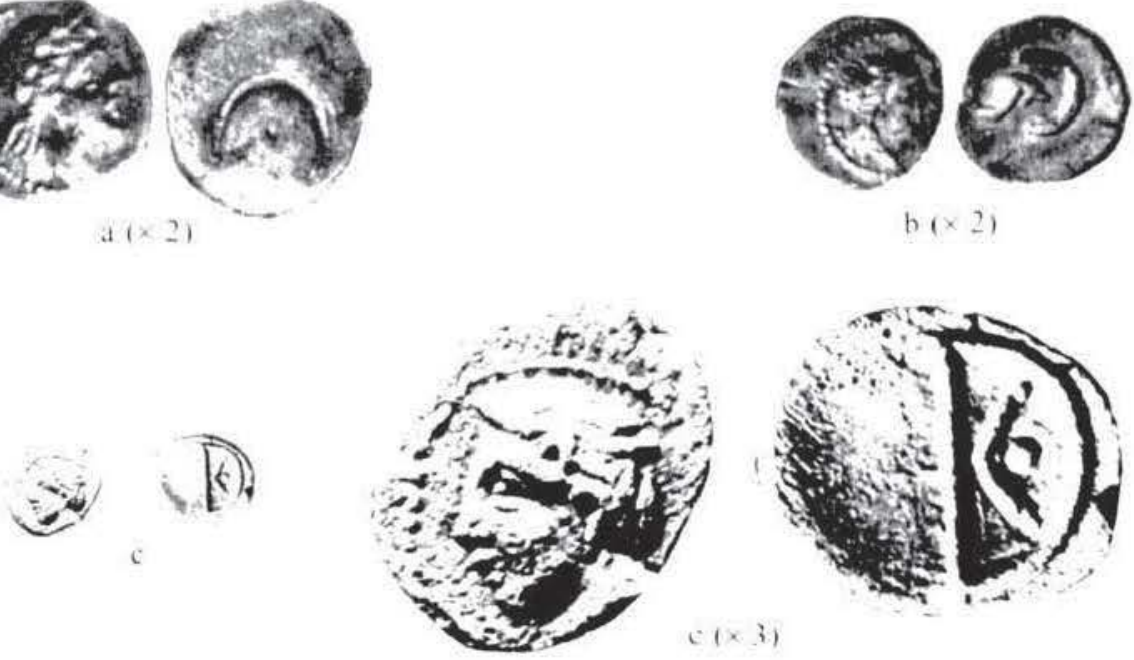

$b(\times 2)$ a $(\times 2)$
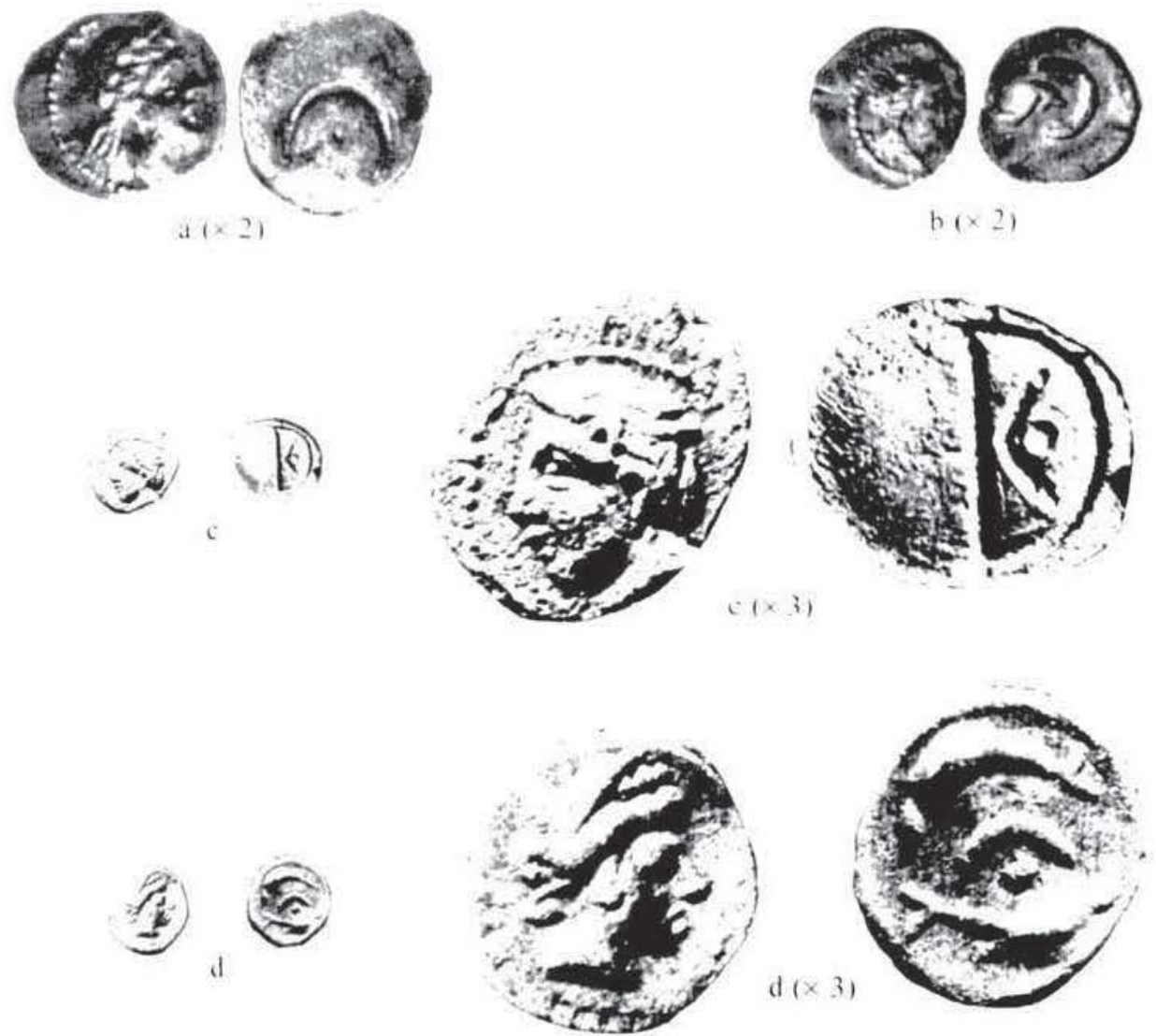

Figura $x$. Pequeños divisores de plata con ereciente.

Roma en 217-215 a. C., en denarios de L. Lucretius Trio del 76 a. C. y en áureos y denarios de P. Clodius del 42 a. C. ${ }^{5}$. Por último, el tipo de reverso de tres crecientes con estrella de 12 rayos dentro de cada uno es habitual en algunos cuadrantes de Venusia y los tres crecientes con glóbulos en semiuncias de Caelia, en monedas de Tarento ${ }^{57} y$ de Crotono $^{5 x}$.

6. M. H. Crawford: Roman Republican Coinage. Cambridge, 1976, n." 39/4, n." 390/1 y 494/20.

Silloge Nummorum Graecorum. The Collection of the American Numismatic Societs. 1. 1969, nums. 755-765. 678 y $1582-1595$.

"Silloge Nummorum Graecorum. The Collection of the American Numismatic Society, 3, 1975, nums 444-446. Fste tipo también aparece en otras acunaciones más lejanas del mundo griego como Eucarpia en Frigia. Byzantium en Tracia, Uranopolis en Macedonia, Cydonia en Creta, Atenas. Sigeium en Troas, Thespiae en Boecia, en algunas monedas partas y en piezas imperiales de Solandus en L.ydia, Alejandria y Carrhe en Mesopotamia.
El creciente junto con el disco solar, la luna y las estrellas, motivos procedentes de antiguos cultos orientales, son atributos de Tanit y Baal Hammon como divinidades astrales que figuran en abundantes manifestaciones religiosas cartaginesas. Su elección en esta ceca parece estar en relación con el posible culto a la diosa, a la que sabemos se veneraba en la Baria púnica $^{54}$. La asociación de la palmera y el creciente con astro también parece tener un simbolismo funerario, como se interpreta en las pinturas de Kef-el-Blida ${ }^{6 i}$.

En conclusión, vemos que tanto la palmera como el creciente, tipos elegidos para las mone-

59 M. J. Almagro Gorbea: Un depósito votivo de terracotas de Villaricos, Homenaje al Prof. Martin Almagro Basch, II, Madrid, 1983, pags, 291-307.

to J. Ferrón: La peinture funeraire de Kef-el Blida, Ar. cheologia. 20, 1968, págs, 52-55. 
das de la Tagilis púnica, se utilizan en la Península solamente en acuñaciones que se circunscriben a la zona mediterránea y son frecuentes en emisiones cartaginesas y norteafricanas.

En cuanto a la leyenda de estas monedas, que transcribimos en TGYLT, como en el caso anterior vemos que se trata de una escritura normalizada que, en sus signos visibles, no presenta problemas de lectura (figura 9). El primer signo es una clara letra taw neopúnica, similar a la inicial de las monedas que forman el tipo 1.

\section{$r / 2 \lambda r$}

Figura 9. Leyenda de las monedas del tipo 2: TGYLT.

La segunda letra es también una clara gimel neopúnica que, como única diferencia con la de las monedas del tipo 1, presenta el trazo derecho prolongado en su parte superior, sobresaliendo del ángulo a manera de ápice ${ }^{61}$. La grafia de esta letra es también parecida a la shin neopúnica de algunas monedas de Shemesh ${ }^{62}$, Asido y otras cecas convencionalmente denominadas «libiofenices», aunque también es similar a la waw que aparece en una rara emisión de Malaca ${ }^{63}$.

El tercer signo es una yod neopúnica similar a la que encontramos en algunas monedas de Ebusus. Esta letra puede ser una mater lectionis con función de vocal, típica del neopúnico, que representa un intento de vocalización respecto a la leyenda de las monedas del tipo 1 .

La cuarta letra es una lamed neopúnica similar a la de la segunda moneda del tipo 1. Por último, en las monedas $n .^{\circ} 2$ y 3 nos parece ver un quinto signo que podría ser una letra nun o una taw, probablemente esta última por comparación con las monedas del tipo 1. En la mone-

\footnotetext{
6) Mazard, pág. 6. Friedrich, n. ${ }^{\circ} 46$.

62 J. Marion: Les monnaies de Shemesh et des villes autonomes de Maurétanie tingitane au Musée Louis Chatelain a Rabat, Antiquités africaines, 6, 1972, pág. 69 , número 22

ה3 Alfaro Asins, 1991, págs. 122 y 130.
}

da $n .^{\circ} 3$ aún parece que pudiera continuar la leyenda pues se aprecian restos de posibles signos apenas perceptibles.

Las consonantes TGL que forman la leyenda, quizás puedan estar en relación con «descubrir o revelar" ${ }^{\text {6-1 }}$, como hipotética alusión a las minas de la zona o incluso al manantial termal de Cela.

En conclusión, lo que parece evidente después del análisis de los dos tipos de monedas que atribuimos a esta nueva ceca púnica es que las dos series que presentamos no parecen ser contemporáneas entre sí, pues difieren en tipos, pesos y especialmente presentan una leyenda en distinto estadio evolutivo. Asi en las monedas del primer tipo algunas letras adoptan aún una forma púnica y todos los signos son consonánticos frente a las del segundo tipo, cuyas letras son todas neopúnicas, quizás algo más evolucionadas, y se introduce una yod con intención de vocalizar el nombre de la ciudad, lo cual es el único argumento que poseemos para obtener una cronología relativa de las monedas entre si.

En este sentido, encontramos un paralelismo entre los dos tipos de leyenda que documentamos en las monedas de esta ceca y las de Tingis. En la ceca africana también hallamos una leyenda en su forma reducida TNG' y otra en su forma plena TYNG' ${ }^{65}$, aunque este criterio epigráfico no parece tener carácter diferenciador para la ordenación cronológica de las monedas $^{66}$.

\section{LA CIUDAD DE TGYLT - RES PUBLICA TAGILITANA}

En cuanto a la ceca que emitió estas monedas, en un principio pensamos que los rótulos TGLT o TGYLT, podian corresponderse con

is M. J. Fuentes: Vocabulario fenicio, Barcelona, 1980. pág. 246 y pág. 97 .

is Mazard, págs. 222-223, leyendas n. 70 y 78 . J. M. Solá Solé: Los rótulos monetarios púnicos de Numidia y Mauritania, Numisma, 35, 1958, págs. 22-23.

ob A. Beltrán: Las monedas de Tingi y los problemas arqueológicos que su estudio plantea, Numario Hispánico, 1952, I, págs. 89-114. Mazard, págs. 180-184, números 589-611. SNGCop, nủms. 720-741. 


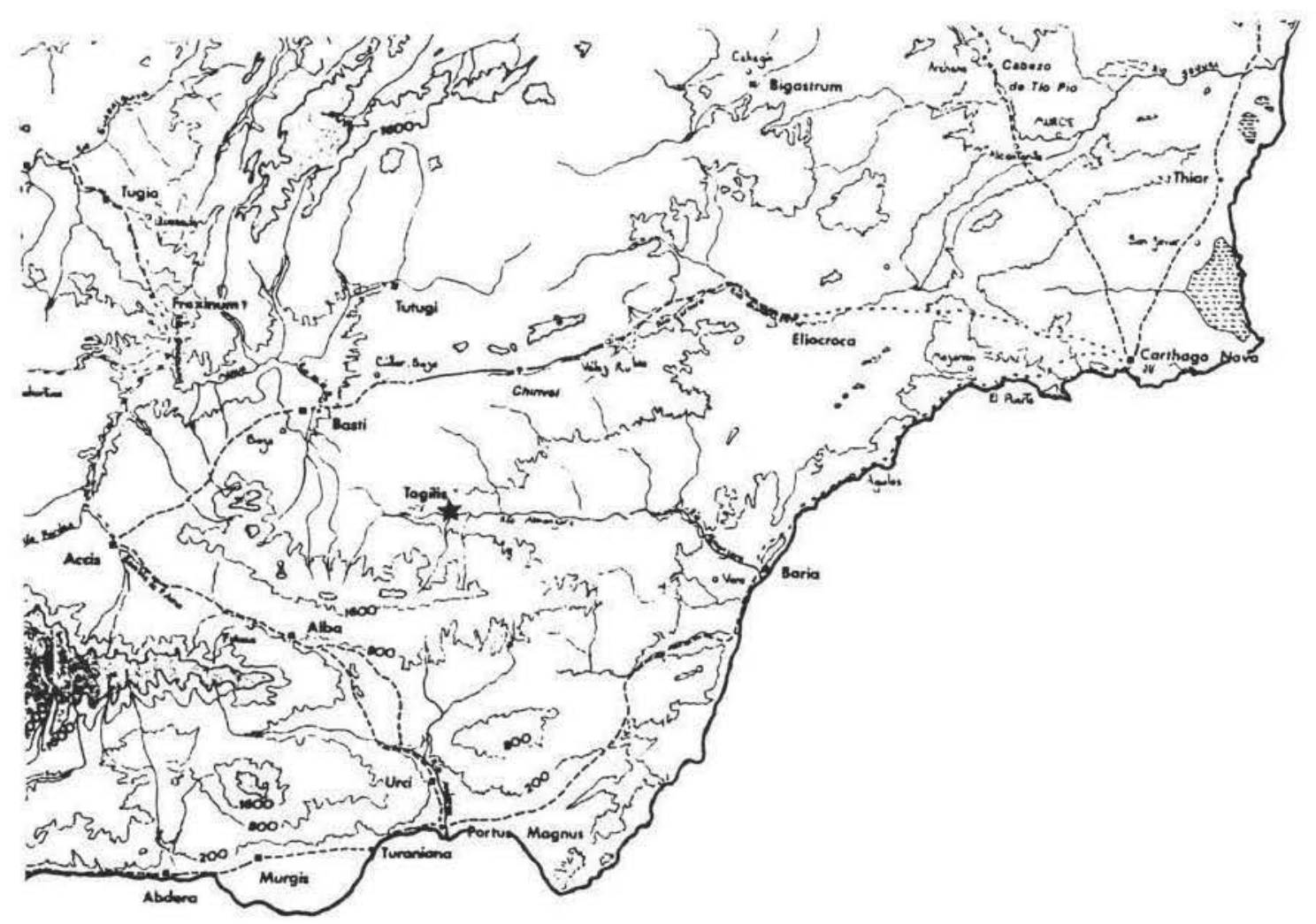

Figura 10.- Mapa de situación de Tagilit/Tagilis (según Sillières).

el auténtico nombre púnico de la actual Villaricos, ciudad que a raiz de la conquista romana por Escipión en el 209 a. C. o algo después, pudo haber perdido el topónimo púnico para tomar el de Baria, nombre latino con el que la citan, entre otros, Cicerón, Ptolomeo y Plinio ${ }^{67}$.

Esta hipótesis inicial de atribución de las monedas a Villaricos se desvaneció ante la evidencia de que su topónimo monetal que transcribimos en TGLT o TGYLT, y que podría vocalizar en TaGYLaT o TaGYLiT, se hallaba documentado en una inscripción romana de cuidada ejecución grabada en una lápida de mármol blanco de 91,50 por $60 \mathrm{~cm}$, aparecida

๑? J. A. Tapia Garrido: Historia General de Almeria y su provincia. Tomo II. Colonizaciones, Almeria, 1982, pág. 304. Según J. M. Solá Solé: Toponimia fenicio-púnica, Enciclopedia Lingüistica Hispánica, I, Madrid, 1960. pág. 499, es muy poco probable una etimología feniciopúnica para este topónimo de Baria. en marzo de 1977 en el término municipal de Tíjola (Almería), que, según Resina y Pastor, dice:

Voconia Q(uinti) f(ilia) Avita

thermas rei publicae

suae Tagilitanae s(olo) s(uo) s(ua) p(ecunia)

$f($ ecit $)$

easdemq(ue) circensibus

editis et epulo dato dedicavit

ad quod opus tuendum usumq(ue)

perpetuum $(t)$ hermarum praeben

dum r(ei) p(ublicae) Tagilitanae denariorum duo milia quingentos dedit

«Voconia Avita hija de Quinto, construyó unas termas en terrenos de su propiedad para la República Tagilitana. Las dedicó después de haber ofrecido un banquete y celebrado unos juegos circenses. Para la conservación y mantenimiento perpetuo de las termas entregó a la 
República Tagilitana la cantidad de dos mil quinientos denarios de platan ${ }^{n \times}$.

En efecto, en esta lápida de mármol de Macael de finales del siglo I, o principios del II d. C. se cita un nuevo topónimo, desconocido hasta ese momento, TAGILITANA, que alude al municipio romano de TAGILI o TAGILIS, cuyo nombre, por el extraordinario parecido fonético, vemos se ha perpetuado en la actual localidad de Tíjola, donde se halló la pieza, situada a unos $60 \mathrm{kms}$. de Baria. (Figura 10.)

$\mathrm{Al}$ parecer son muy abundantes los hallazgos arqueológicos de la zona de Tíjola, donde hay constancia de varios yacimientos arqueológicos desde el paleolítico a época moderna. Desde el punto de vista de las monedas que presentamos, destaca una enorme factoría púnica emplazada en la Muela del Ajo, cerro de contorno irregular situado a unos $1.800 \mathrm{~m}$. al norte-noroeste de Tijola, en la margen izquierda del río Almanzora.

Se trata, según Pellicer y Acosta, de un gran núcleo púnico industrial y comercial de los siglos VI-III a. C., formado por la penetración de gentes púnicas venidas de Baria que ascenderían por el valle de Almanzora en busca de minerales de cobre (Cueva de la Paloma), hiero (Serón) y mercurio (Bayarque). Además otros atractivos de la zona serían tambièi su riqueza agrícola y el manantial natural de Cela. La superficie del yacimientos de unos $100.000 \mathrm{~m}^{2}$, según los citados autores, está repleta de fragmentos cerámicos, predominando las ánforas púnicas con formas arcaicas y los grandes vasos de la misma tradición. También abundan las escorias de hierro, plomo y cobre. En la zona igualmente hay constancia de otros yacimien-

${ }^{n}$ x P. Resina Sola, y M. Pastor Muñoz: Inscripción romana aparecida en Armuña del Almanzora (Almeria), Zephyrus, XXVIII-XXIX. 1978, págs. 333-336. R. Lázaro Pérez: Inscripciones romanas de Almeria, Almeria, 1980 págs. 91-93. En la zona de Tijola han aparecido también recientemente, 1978 y 1979 , otras inscripciones latinas de tipo funerario. Cfr. R. Lázaro: Municipios romanos de Almeria. (Fuentes literarias y epigráficas.) I Encuentro de Cultura Mediterránea. Homenaje al Padre Tapia, Almeria. (1986), 1988, pág. 124. Hispania Epigraphica, 2, 1990, pág. 22, n. ${ }^{\circ}$ 23. P. Resina: Inscripción romana aparecida en Tíjola (Almeria), Zephyrus, 32-33, 1981, 213-215.

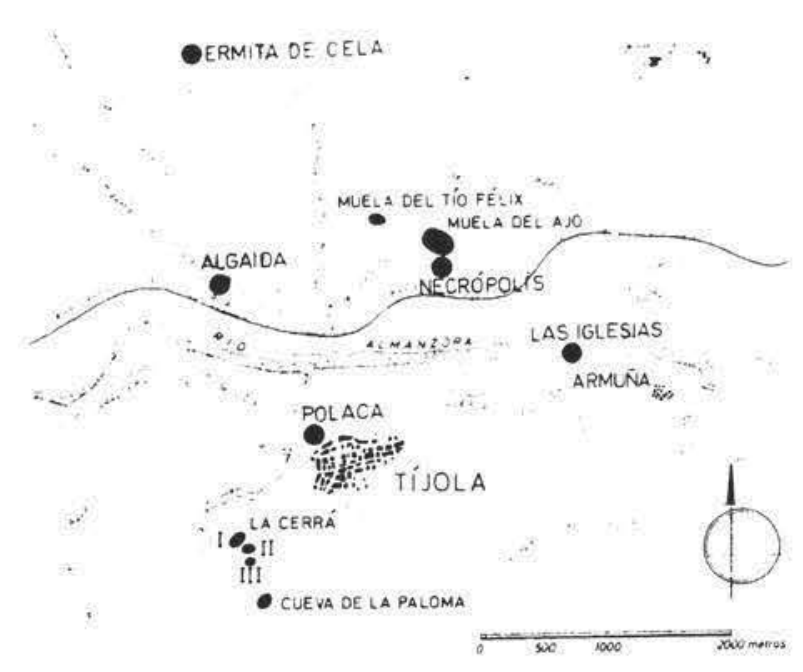

Figura I1. Plano de la zona de Tijola (según PellicerAcosta).

tos romanos posteriores, desde finales del siglo III a. C., de tipo eminentemente minero e industrial ${ }^{69}$.

Esta factoria púnica tierra adentro ${ }^{71}$ era paso obligado desde el Mediterráneo a la altiplanicie granadina que conducía a la zona minera de Jaén. La vía, jalonada de yacimientos arqueológicos de todas las épocas, unía desde antiguo los centros mineros donde se extraía el oro de Sierra Nevada y el plomo argentífero de Sierra Mórena, con Baria, puerto de embarque del mineral. La cuenca del Almanzora y del Guadiana Menor fue probablemente el camino más utilizado para las relaciones entre el litoral del Levante mediterráneo y el alto Valle del,Gua-

(19) M. Pellicer, y P. Acosta: Prospecciones Arqueológicas en el Alto Valle del Almanzora (Almeria), Zephyrus, XXV, 1974, pảgs. 155-176. También se señalan otras prospecciones más recientes en $P$. Cressier: Prospección arqueológica en la sierra de los. Filabres y el alto valle del Almanzora (Almería), 1985, Anuario Arqueológico de Andalucia, 1985, Sevilla, 1987, pág. 73, U. P. Cressier: Segunda campaña de prospección arqueológica en la sierra de los Filabres y el alto valle del Almanzora (Almería), Anuario Arqueológico de Andalucia, 1986, Sevilla, 1988. pág. $113, \mathrm{n} .^{\circ} 14$.

70 El fenómeno colonizador semita bien pudiera ser que no tuviera un carácter tan marcadamente litoral: sus vestigios empiezan a encontrarse a más de $150 \mathrm{Kms}$. de la costa, como han señalado J. Fortea, y J. Bernier: Recintos y fortificaciones ibéricos en la Bética, Salamanca, 1970 , pág. 133. 


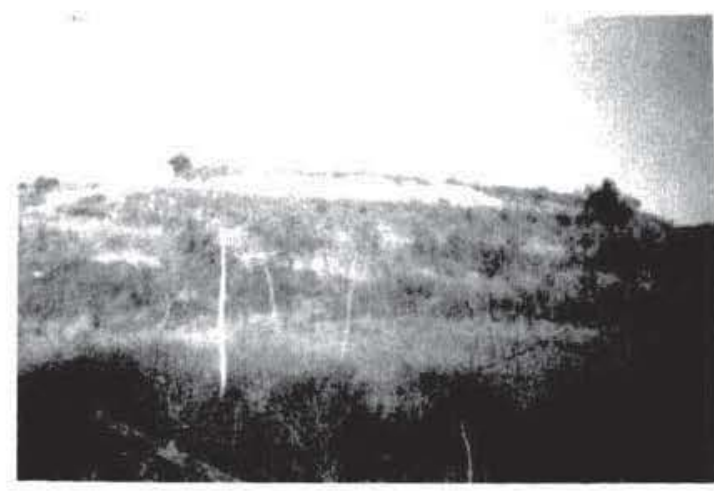

Figura 12. Vista de la Mucla del Ajo de Tijola.

dalquivir. Esta antigua via de penetración. utilizada ya en época argárica, partiendo de $\mathrm{Ba}$ ria, remontaba el valle del rio Almanzora, pasaba por Tagilis, Basti, Castellones de Ceal, Tugia, Ubeda la Vieja, Ibros y finalmente llegaba a Cástulo, desde donde continuaba hacia otras direcciones ${ }^{71}$.

Esta via, de principio a fin, está jalonada de ricas necropolis y yacimientos en los que se ha constatado una fuerte presencia de cerámicas griegas de importación. Asi en la Muela del Ajo de Tijola, donde los materiales muestran una ocupación desde el siglo $\mathrm{v}$ al II a. C., según Rouillard, se hallo un fragmento de pie de crátera de figuras rojas datado en la primera mitad del siglo iv a. C. También en la mina de cobre de la Cueva de la PaIoma, situada $800 \mathrm{~m}$ al sur de Tijola la Vieja, una prospección de C. Domergue ha proporcionado materiales que permiten datar la explotación entre principios del siglo IV a. C. y el siglo vi d. C., entre éstos un fragmento de cerámica ática de barniz negro fechado entre 380 330 a. C. ${ }^{72}$.

Estamos pues ante las monedas de la factoria púnica de Tagilit o Tagilis que debió ser to suficientemente próspera como para importar

\footnotetext{
"P. Sillières: Les voies de communication de l'Hispanic Meridionale, Paris, 1990, págs. 552-553

12 P. Rouillard: Les grees et la Péninsule laerique du Whe au the siêcle avant Jésus-Christ, Paris, 1991, páginas 125 y $658-659$
}

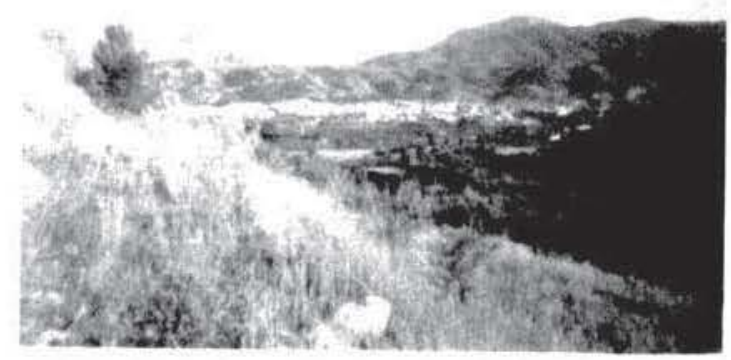

Figura 13. Vista de Tijola desde la Muela del Ajo.

cerámicas griegas y acuñar moneda. De esta ciudad no queda más testimonio de su pasado púnico que los fragmentos cerámicos que citan Pellicer-Acosta y estas monedas que no sólo nos proporcionan el testimonio de una ciudad púnica con complejidad politica suficiente para acunar moneda, sino también su auténtico nombre púnico, topónimo que permanece inalterado en época romana, como vemos por la inscripción de época flavia de Voconia Avita.

No sabemos qué suerte corrió la ciudad ante la conquista romana, si fue tomada por la fuerza como Baria en el 209 a. C., o se sometió libremente como las restantes cecas púnicas. Probablemente la segunda opción está más de acuerdo con la permanencia del topónimo púnico de la ciudad en época romana.

La población púnica de esta zona debió estar bastante iberizada o, al menos, muy en contacto con gentes ibéricas como se ve también en Villaricos, ciudad que estaba en el limite de lo que era Ptolomeo $(I I, 4,6)$ eran los «bástulos llamados púnicos» que habitaban desde el Estrecho hasta Baria. También se trata de una zona muy romanizada después, próxima a la ciudad de Acci (Guadix) que albergó a los veteranos de dos legiones de César, que entre los años 7 y 2 a. C. pasó a formar parte de la Tarraconense.

La ciudad romana de Tagilis también debió tener cierta importancia pues tenia termas 
junto al citado manantial salutifero de Cela, un bosque sagrado y celebraba juegos circenses, según la citada inscripción, aunque su nombre no figura en ninguna fuente latina. La primera vez que se cita este topónimo es en El Idrisi. leido por Saavedra como Torchela y Tâyula, de donde, al parecer, procede el nombre actual de Tijola ${ }^{73}$.

R. Láaro Pére: Inseripclones romanas de Almeria. citado, págs. 10-20. A. Tovar: Iberische Landeskunde. Tarraconensis. Baden-Baden. 1989, paigs. 159-160.

\title{
PRIMER AVANCE SOBRE EL GRAN BRONCE CELTIBÉRICO DE CONTREBIA BELAISCA (BOTORRITA, ZARAGOZA)
}

POR

\author{
MARÍA ANTONIA DÍAZ SANZ
}

Directora de la excavación arqueológica

Y

\section{MANUEL M. MEDRANO MARQUÉS}

Departamento de Arqueologia de la Universidad de Zaragoza

\section{RESUMEN}

Se presenta en este trabajo una breve descripción arqueológica y cronológica del yacimiento de Contrebia Belaisca, incidiendo especialmente en los datos proporcionados por las campañas más recientes de excavaciones. Se ofrece una visión global del entorno histórico en que se encuadran los tres documentos escritos sobre bronce hallados en la ciudad, aportando nuevos datos arqueológicos sobre el hallazgo del primer texto celtibérico. El objetivo primordial es dar un avance sobre el gran bronce de Botorrita, aparecido en la campaña de excavaciones de 1992, comentando los datos que proporcionan las circunstancias de su descubrimiento y la lectura de algunos signos que se observaban a simple vista, antes de su limpieza.
SUMMARY

This paper consists of a brief archaelogical and chronological description of the site-find of Contrebia Belaisca, with special emphasis on the data obtained from the most recent excavations. A general view of the historical background in which the documents written on bronze found in the city is given, and new archaelogical data about the finding of the first Celtiberian text are offered. The principal objective is to make a preliminary study of the large bronze found in Botorrita during excavations in 1992, commenting on the data which its discovery provided and the reading of some signs which were seen prior to its cleaning. 